\title{
LA PERTINENCIA COMO REQUISITO PARA LA CALIDAD EN EDUCACIÓN SUPERIOR. LA PLANEACIÓN INSTITUCIONAL Y EL COMPROMISO COMO CONDICIÓN ESENCIAL PARA EL DESARROLLO DEL POSGRADO
}

Elvia Méndez Fregozo

Catedrática de la Escuela de Humanidades, Universidad Autónoma de Baja California

\section{PRESENTACIÓN}

La urgencia de no postergar más la construcción de respuestas holísticas ${ }^{1}$ que atiendan las problemáticas nacionales a fondo y de manera integral -a partir de la definición de su importancia local, pero con la clara noción de su vínculo con el sistema mundial-, obliga a las instituciones de educación superior a situar en la mesa de discusiones el sentido de sus aportaciones, así como el alcance de aquellas propuestas hechas desde la perspectiva de la innovación de los procesos para el desarrollo de sus funciones sustantivas.

Cuestiones como el crecimiento acelerado de la pobreza, el detrimento ecológico -manifiesto en la desertificación y la contaminación vertiginosa y el aumento exponencial de la violencia social- desde el uso legítimo de la violencia por parte de las instituciones, las manifestaciones primarias de delincuencia, son sólo algunos de los aspectos que destacan en el escenario contemporáneo. ${ }^{2}$

Ante el escenario descrito, es posible enunciar que muchas de las organizaciones sociales se ven superadas frente a dichos desafíos; y no es que no se perciban las necesidades y se atiendan, el punto es que la velocidad y lo inédito de las situaciones emergentes no han dado margen para construir marcos integrales que permitan comprender y resolver las nuevas exigencias; los contextos son nuevos y complejos, $y$ las respuestas son casi las mismas: parciales, simples y de corto plazo.

La incorporación de los retos al sentido de funcionamiento de las organizaciones sociales posibilita tener una percepción más clara del papel que desempeñan éstas en la coyuntura actual; y, lo que es más trascendente, comprender la función que tendrán en el futuro mediato y en un horizonte más lejano de acción.

En este contexto este escrito presenta una reflexión en torno a una propuesta de actuación de las Instituciones de Educación Superior (IES), en particular acerca de las acciones a considerar en su desempeño en escenarios futuros que deparan una mayor incertidumbre. En este caso, se abordan los niveles de educación superior, de manera específica los estudios de posgrado, ya que en ellos es más visible la centralización del esfuerzo por la formación y actualización de individuos que contribuyan al desarrollo del conocimiento validado. Es cecir, en estos espacios educativos se construyen y reproducen los marcos referenciales de ciencia y tecnología, que a su vez sirven para aprobar la noción aceptada de vida y realidad.

\footnotetext{
1 UNESCO (2000) p. 7.

${ }^{2}$ UNESCO (2002) pp. 34-59
} 
Es necesario destacar que la tarea de las IES las trasciende, es decir, las actividades que desarrollan van más allá de su propia existencia, o del desarrollo eficiente de sus funciones. Este tipo de organizaciones tanto públicas como particulares- usufructúan una condición de privilegio debido a que la sociedad ha depositado una confianza total en que allí se elaboren, generen y divulguen percepciones y nociones aceptadas por el conjunto social sin mayores cuestionamientos. Esta situación no sólo les permite reproducir su existencia y su permanencia en los espacios sociales, sino acrecentar día a día su presencia con el consabido peso y validez de sus aportaciones al colectivo social.

Ante esta circunstancia se plantea una serie de interrogantes para guiar la reflexión: ¿son prescindibles las IES en los escenarios futuros de México?, ¿la formación de cuadros de alto nivel es la estrategia adecuada para la transformación cualitativa de las condiciones de vida de la sociedad contemporánea?, ¿un sistema educativo, altamente condicionado, puede elaborar rutas de emergencia para los problemas urgentes señalados anteriormente?, ¿ante la situación explícita donde las IES han sido rebasadas, puede construirse una posibilidad de acción?

\section{GESTIÓN EDUCATIVA Y PLANEACIÓN PROSPECTIVA COMO INSTRUMENTO DE ORGANIZACIÓN ACADÉMICA INTERNA}

Es posible mencionar la ambigüedad en el uso de los fines con respecto a los medios para la resolución de un problema: al utilizar un esquema lineal se anteponen los medios a los fines, con el argumento de que la cantidad o calidad de los procedimientos necesariamente se verá reflejada en los resultados del proceso.

Por otra parte, se trazan objetivos donde no se presta mayor atención a la manera en que se obtendrán; ambas situaciones -aunque aparentemente polarizadas- guardan una gran semejanza por el proceso de diseño de la organización académica interna que elabora el programa de posgrado. Es necesario establecer con claridad los conceptos guías para la relación entre el programa de posgrado y la organización académica y administrativa seleccionada para sustentarlo.

Las labores de cualquier organización requieren de acciones eficientes y eficaces para el desempeño de sus funciones. Al establecer su misión, cada institución determina el futuro que desea para sí y en consecuencia establece los medios adecuados para construir esa noción deseada para el futuro. Se da entonces, el proceso de toma de decisiones que corresponda a las estrategias para así cumplir los objetivos deseados para la organización educativa, y -lo que es más relevante- que sean fines pertinentes a las necesidades establecidas por los entornos donde se desempeña cada espacio educacional.

Es así que las tareas de gestión conllevan una acuciosa labor de planeación, entendida ésta en toda su complejidad como:

[...] un proceso continuo y sistemático, en el cual se aplican y coordinan los métodos de investigación social, los principios y las técnicas de educación, de la administración, de la economía y de las finanzas, con la participación y el apoyo de la opinión publica, tanto en el campo de las actividades estatales como privadas, a fin de garantizar educación adecuada a la población, con metas y etapas bien determinadas, facilitando a cada individuo, la realización de sus potencialidades, y su contribución más eficaz al desarrollo social y económico del país. ${ }^{3}$

\footnotetext{
${ }^{3}$ LATAPI Apud ÁLVAREZ (1982)
} 
En la planeación se establecen los siguientes puntos para su acción: diagnóstico, objetivos, temporalidad y decisiones pertinentes. Se trata de que la gestión estratégica, como intermediación, logre el cambio planeado, y lleve a la organización desde la situación actual hasta una final que corresponda a la visión y a la misión construidas con anticipación.

Para realizar este tipo de tareas se han establecido una serie de criterios que, según Sanders, ${ }^{4}$ son: eficiencia, eficacia, pertinencia y relevancia. En este ensayo lo que se busca es destacar que, si bien tres de ellos exigen atención en el presente -ya que de ellos dependen los resultados a obtener-, en el caso del criterio de pertinencia, éste debe atender otras temporalidades; es decir, es imprescindible incluir el futuro y el pasado en la definición del concepto de pertinencia en las labores de la organización.

Un punto que resulta destacable es la consideración de que la planeación guarda una relación estrecha con el entorno, dado su carácter político, el cual destaca que "con frecuencia suele pensarse que la planeación es una actividad puramente técnica, objetiva, y neutral, desde el punto de vista cultural, moral y político; pero en los hechos, la planeación constituye un proceso complejo y multidimensional que dista mucho de la neutralidad y objetividad que se le atribuye a la ciencia". ${ }^{5}$

Ahora bien, según la UNESCO, el concepto de pertinencia en educación superior atiende a lo siguiente:

La pertinencia en educación superior se considera primordialmente en función de su cometido y su puesto en la sociedad, de sus funciones con respecto a la enseñanza, la investigación y los servicios conexos, y de sus nexos con el mundo del trabajo en sentido amplio, con el estado y la financiación publica y sus interacciones con otros niveles y formas de la educación. ${ }^{6}$

Específicamente, la definición de planeación para este nivel educativo se entiende como: "un conjunto de procesos coordinados, sistemáticos y generalizados para la determinación de acciones tendientes al desarrollo equilibrado y coherente de la educación." 7 De ahí que pueda categorizarse como una actividad racional, integral y permanente que a partir del interés de las organizaciones llega a formar parte nodal de su desempeño. Asimismo, tal actividad apoya la toma de decisiones ya que perfila futuros posibles con las consabidas acciones necesarias a desarrollar. $Y$ en última instancia hace más eficiente todo el proceso de gestión ya que permite que los recursos disponibles se empleen mejor.

El empleo eficiente y adecuado de los recursos existentes en cualquier organización exige la presencia y el uso de la planeación como un auxiliar en la toma de decisiones. Empero, en el ámbito educativo es condición imprescindible definir con claridad las características y el sentido que la planeación tendrá. En lo que respecta al escenario de México, el trabajo de planeación surgió con el argumento de que debía apoyar el desarrollo del sistema de educación nacional, aunque se arraigó y evolucionó inicialmente en el nivel de educación superior, sobre todo por el hecho de que había "crecido vertiginosa y asombrosamente" durante la década de los años setenta, debido a políticas populistas de un gobierno federal que estuvo dispuesto a congraciarse con este nivel educativo. ${ }^{8}$

\footnotetext{
${ }^{4}$ SANDER, B.( 1982) p.9-21

${ }^{5}$ ALVAREZ, I. (1982) p. 183-203

${ }^{6}$ UNESCO (1998) p. 19

${ }^{7}$ ANUIES (1979). p. 25

${ }^{8}$ ANUIES (2000).
} 
En retrospectiva, es posible apreciar que el instrumento técnico de la planeación se convirtió en un fin en sí mismo, ya que la toma de decisiones seguió apartada de las propuestas de planeación y vinculada a coyunturas sociales y políticas. Sólo fue hasta épocas muy recientes -específicamente en la década de los noventa y forzados por restricciones financieras- que se desplegó una estrategia nacional en el nivel superior público para atender, entre otros aspectos, los siguientes: oferta educativa, formación y contratación de planta docente y modernización de los sistemas administrativos.

Cabe mencionar que en la primer época de la planeación, en los setenta, no hubo responsabilidad del incumplimiento debido a las cuestiones técnicas. Puede decirse que la planeación no consiguió impactar ni desplegar a fondo sus alcances y posibilidades porque significaba generar compromisos y, consecuentemente, crear expectativas que en la realidad no se buscaba cumplir. Fue durante ese periodo que se creó un sistema para la planeación del nivel educativo. Para respaldar estas tareas surgió la Comisión Nacional para la Planeación de la Educación Superior (CONPES), la cual concibió una infraestructura con alcance nacional, regional (CORPES), estatal (COEPES), e incluso a nivel del aparato de cada institución (unidades institucionales de planeación).

Las autoridades educativas del nivel superior han trazado políticas donde ya se vislumbra la planeación como el medio a través del cual es posible forjar los esfuerzos iniciales para conocer, ordenar y diseñar un futuro posible para cada organización educativa; además, se concibe como un esfuerzo colectivo imprescindible en el que deben participar todos los actores e incluirse todas las dimensiones que conforman la vida humana asociada; y, finalmente, considerar las variables derivadas de procesos que condicionan el desarrollo de la educación superior. Es importante mencionar que en el inicio, la tarea de orientar al nivel superior correspondió a la licenciatura, y hasta años muy recientes se construyó un esfuerzo de planeación del posgrado a nivel nacional..$^{9}$ Es ahí donde se enfoca el interés del presente ejercicio de reflexión, con la inquietud de brindar elementos que permitan que la formación y actualización de los estudiantes de posgrado se realice sobre bases de una acción que atienda criterios trascendentes y no tan sólo a los de coyuntura; los tiempos y la situación social así lo exigen. ${ }^{10}$

En la actualidad se considera que la función de la planeación institucional es un instrumento imprescindible para cada centro educativo, pues brinda la posibilidad de erigir condiciones idóneas para operar bajo la óptica de la transformación constante y la atención adecuada a las necesidades del entorno. Para el caso del posgrado, la planeación resulta estratégica por la trascendencia e impacto que significa la actividad de formación y actualización de personal especializado de alto nivel, el cual -en muchos casos- será el responsable de la generación y divulgación del conocimiento científico en el país. En esto último, se encuentran depositadas las posibilidades del futuro, pero también la realidad del presente.

\section{LA CONSTRUCCIÓN DEL CONCEPTO DE PERTINENCIA SOCIAL.}

La sociedad encomienda a sus organizaciones múltiples funciones y les otorga el sentido que requiera la coyuntura sociohistórica. En el caso de las organizaciones educativas, la asignación del compromiso histórico de contribución se da desde su origen. Así, la educación formal ha recibido la tarea de aportar de

\footnotetext{
${ }^{9}$ CONACYT (2001)

${ }^{10}$ UNESCO (2002) p.34-59
} 
diversas maneras a su entorno, unas veces para formar ideológicamente, otras para modelar las competencias que exige el mercado laboral.

Desde esta perspectiva, la idea del vínculo entre los espacios educativos y su medio ambiente siempre ha estado presente, unas veces de manera implícita, otras de forma explícita. En el inicio del siglo XXI, el contexto de los sistemas educativos exige a los miembros que los integran dos condiciones esenciales: primero, vigilancia para la imprevisibilidad, dada la enorme inestabilidad del mundo globalizado; y segundo, una marcada pertinencia de los resultados de sus acciones. En esta última circunstancia es donde se ubica la reflexión de este documento: a la creciente exigencia que el entorno plantea a las organizaciones educativas, con el argumento de que es la sociedad la que contribuye con su esfuerzo (financiamiento), y por ello a la que habrá de dársele cuentas. Es necesario anotar que el concepto de pertinencia es una construcción sociohistórica, y responde directamente a los esquemas de desarrollo de la sociedad en que se inserta cada organización educativa. Además, como todas las actividades de edificación de la idea del mundo, el concepto de calidad también deviene de un proceso social.

En la mayoría de las instituciones se evalúa el rendimiento académico o el suceso de un programa de posgrado con base en esquemas ajenos, lo que deviene en una evaluación desventajosa para aquellos que se encuentran en condiciones desiguales de desarrollo. Tal situación acarrea, a su vez, restricciones de prestigio y de apoyo financiero, lo que crea obstáculos infranqueables en la evolución general de este nivel de estudios. Si se hace esta consideración, puede conformarse una política nacional que, con apoyo en una perspectiva adecuada, posibilite los avances del posgrado en cada universidad pública bajo esquemas de calidad y pertinencia.

No es que se niegue la necesidad de conocer cuál ha sido, y es en este momento, la actuación de una universidad; ello es necesario e indispensable para validar socialmente los esquemas, las metodologías y los fines de la acción institucional. Lo que se aduce es que, en primera instancia, debe considerarse la participación efectiva y real de la comunidad universitaria para elaborar un modelo de evaluación. El proceso de participación -en su infinidad de variantes-abre las posibilidades de adhesión a un proyecto, y en el caso del desarrollo del posgrado -bajo criterios de calidad y pertinencia- es necesario aceptar los puntos de referencia de la institución.

La consideración de las diversidades regionales -con sus valores específicos- es una cuestión imprescindible para construir un esquema de planeación y evaluación de los estudios de posgrado. Allí se encuentra la clave para sustentar la pertinencia.

Una de las primeras preocupaciones latentes es la necesidad de un marco valorativo, sin el cual no podría hablarse de prioridades de ninguna especie; porque, precisamente, las prioridades implican una jerarquización y la jerarquización implica un criterio para jerarquizar. Este criterio no es otra cosa que la opinión sobre lo que vale más o menos la pena en el momento histórico y en el contexto social de la región. Esto de lo que vale más la pena es un juicio de valor que [...] brota de la naturaleza misma del proceso de asignación de prioridades. ${ }^{11}$

Es este sentido, el que posibilita la construcción de un esquema de desarrollo del posgrado bajo criterios de calidad y pertinencia, con la participación consciente de la colectividad y sus responsables.

\footnotetext{
${ }^{11}$ VARIOS (1971) p.134
} 
En este momento histórico de cambios globales en los esquemas generales de ordenación es intrascendente hablar de modernización, de democracia, de participación, de calidad, de pertinencia en los discursos. Lo relevante radica en el sentido del impulso para el establecimiento del posgrado frente a quienes detentan la autoridad educativa nacional; así como en colaborar para atender, en incluir para sumar:

La relevancia, tanto en el estudio como en el ejercicio de la administración, es un concepto filosófico y político, exigiendo por parte del investigador y del administrador, además y por encima del dominio técnico, un compromiso definido con la calidad de la vida humana asociada, que debe ser el propio objeto de la ciencia social y, en ese contexto, de la administración de la educación [...]. ${ }^{12}$

\section{MARCOS DE REFERENCIA PARA EL POSGRADO.}

Uno de los intereses centrales de este trabajo es la reflexión sobre las características generales que las IES han adoptado en México en los años recientes, en lo que se refiere a los estudios de cuarto nivel. Se trata de examinar cuáles son las formas que imperan para su establecimiento, organización y desarrollo. Otro interés es abundar sobre el análisis y discusión acerca del vínculo de la acción de las IES con la categoría de congruencia.

En el contexto sociohistórico contemporáneo es imprescindible revisar el marco discursivo manejado institucionalmente acerca de la calidad y pertinencia de las acciones, contrastado con los indicadores objetivos de eficacia y congruencia que sustentan y orientan el crecimiento y desarrollo de los espacios educativos universitarios. La premura coyuntural en sociedades como la mexicana es la que conlleva la necesidad de repensar los sentidos de las organizaciones educativas, pero, sobre todo, el direccionamiento que se le está dando al nivel superior de estudios, donde se atienden dos criterios: el primero es la cientificidad, establecida por organismos oficiales; el otro, la uniformidad, exigida en los perfiles de formación de las personas que conforman los cuerpos académicos de los posgrados. ${ }^{13}$

Por otra parte, este ejercicio reflexivo busca aportar una noción distinta para la acción organizativa y el funcionamiento de los estudios de posgrado: se trata de una percepción que vincule la calidad con el sentido y la pertinencia; una idea de relación que conlleve la perspectiva holista, donde los requerimientos de calidad y pertinencia en las organizaciones comprometan su funcionamiento en dimensiones sociales más amplias y profundas y no únicamente en el mercado laboral y en los sectores productivos. Se trata de reestablecer el vínculo de las universidades, y sus estudios de cuarto nivel, con el sentido de equidad y justicia social. Sólo una noción así podrá coadyuvar a transformar -en el mediano y largo plazo- las condiciones sociales que muestran un creciente detrimento en la calidad de vida de la sociedad mundializada.

El interés por planificar y desarrollar los estudios de posgrado se origina en un reconocimiento tácito de su potencialidad para la formación de personas para el desarrollo de tareas de investigación, y la estructuración académica de cuadros de alto nivel para la toma de decisiones estratégicas que contribuyan al desenvolvimiento de áreas del conocimiento científico y tecnológico que hasta el momento no han sido desarrolladas o bien presentan un mínimo avance, en México.

\footnotetext{
${ }^{12}$ SANDER, B. (1982) p.28

${ }^{13}$ Se trata de los programas de Posgrado de calidad de Consejo Nacional de Ciencia y Tecnología y el Programa de Mejoramiento del Profesorado de la Subsecretaria de Educación Superior e Investigación Científica de la Secretaria de Educación Pública.
} 
En un escenario nacional donde se presenta una grave carencia de espacios para la generación de conocimientos innovadores, los posgrados representan por excelencia, el espacio educativo con un impacto multiplicador, que deviene en una acción positiva para la búsqueda de respuestas, y para la generación de satisfactores a las necesidades sociales más apremiantes. De igual manera, en estos espacios también pueden generarse los cuerpos académicos -ethos- que, con base en una discusión pertinente, constante y relevante, puedan aportar visiones novedosas que contribuyan a leer la realidad y, a partir de esto, se puedan operar cambios significativos para el resto de las actividades institucionales universitarias. Esto con el fin de hacer más pertinentes las acciones del subsistema de educación superior y del sistema educativo en general $y$, de alguna manera, extender los beneficios al conjunto de la sociedad por medio de la vinculación.

En México es inobjetable que la investigación desarrollada en los espacios universitarios se ha convertido en una de las fuentes primordiales para el avance de la ciencia y la tecnología, así como de indagación humanística. Es en este sentido que, su apoyo e impulso decidido dentro del ámbito de educación superior, resulta un factor esencial para poder configurar el desarrollo de la nación.

Nunca como hoy, en el escenario mundial se ha reconocido la trascendencia e impacto que tienen los nuevos conocimientos en la configuración de las condiciones para el futuro de una sociedad; la producción de ideas relacionadas con el avance del conocimiento humano, lo mismo que la formación de recursos de alto nivel, pueden ser vistos como bases para definir perfiles y niveles de desarrollo social. Percatarse de esto, trae consigo la necesidad de planear, sustentando tal acción en el criterio de pertinencia (Morín, 2003).

Establecer redes y núcleos de formación ligados a centros universitarios -generalmente a través de los programas de posgrado- con carácter formal en sus diversas modalidades, resulta una estrategia de un valor incalculable para el desarrollo del país. Frente a esto, es importante que las condiciones de origen y crecimiento de los programas de posgrado tengan determinadas características, y cumplan con condiciones estipuladas con base en diagnósticos adecuados que muestren la complejidad multidimensional del entorno universitario.

En esta reflexión se busca exponer posibles consecuencias que tienen las acciones improvisadas en este tipo de esfuerzos educativos; así como mostrar que el sustento de la planeación se convierte en una herramienta para determinar el sentido de las acciones educativas del posgrado, en congruencia con su contexto.

\section{LOS MARCOS PARA LA EVALUACIÓN DEL POSGRADO.}

El origen, formas de desarrollo y pautas de evaluación de los estudios de posgrado pueden ser considerados como parámetros que nos permiten conocer el grado de evolución o estancamiento del nivel de educación superior en un país; ello es visible en gran medida porque es ahí donde se distribuyen y generan muchos de los conocimientos más innovadores que ayudan a elevar los niveles de desarrollo en México.

En nuestro país existe una gran cantidad de centros educativos que atienden este nivel de estudios, cada uno de ellos con criterios propios en relación a métodos y contenidos, incluso con visiones particulares en lo que se refiere a organización y fines académicos. Por tanto, llega a constituirse en un sistema heterogéneo, con modelos y tipologías que se subdividen hasta niveles mínimos dentro de una misma 
disciplina y área de conocimiento; también se deriva en un universo cada vez más complejo donde uno de los retos iniciales es conformar un marco de análisis que permita abordarlo en toda su riqueza, con el fin de superar juicios reduccionistas y lineales que resultan en escenarios poco factibles.

Bajo estas circunstancias, cada institución posee perfiles sui generis y problemáticas específicas, las cuales se manifiestan en varios aspectos que abarcan desde: grados de diversidad y complejidad del programa, de sus contenidos hasta el sentido que éste tiene; por otra parte, contribuyen a la fisonomía del programa, las características y niveles de desarrollo de cada institución.

A pesar de la multiplicidad de condiciones y tipos del posgrado, existe una corriente de evaluación dentro de los organismos oficiales, los cuales han establecido una serie de mecanismos y parámetros constituidos en un paradigma general basado en el concepto de calidad (padrón de excelencia); sin embargo, éste resulta constreñido para un universo tan diverso como el que representa la oferta educativa del cuarto nivel.

En este intento de evaluación que busca cierta homologación impera el enfoque de evaluación positiva como un criterio rector para la consecución de apoyos de carácter financiero (becas y apoyos adicionales). Es importante mencionar que esta perspectiva reduce las posibilidades de contribución cualitativa de una valoración, pues hace aparecer a este tipo de acciones como una tarea fiscalizadora y penalizadora, en vez de una etapa de revisión y replanteamiento del posgrado; es decir, como una posibilidad estratégica del desarrollo nacional.

Así, las políticas educativas se ven transformadas en líneas de acción que corren en sentido contrario; o bien, se traducen en serios impedimentos para el desarrollo de instituciones y programas educativos de cuarto nivel, que al no reunir las condiciones establecidas, no pueden contar con los apoyos necesarios para su perfeccionamiento y consolidación final.

Aunque es indiscutible la necesidad de establecer patrones mínimos de calidad para el funcionamiento de los programas, es importante tener en consideración las condiciones de especificidad regional en que éstos surgen. Por ello, se hace fundamental examinar cuidadosamente -de desde la perspectiva de cada institución de educación superior- las propuestas nacionales y sus tendencias, con el fin de sopesar las posibles consecuencias y costos de inclusión; asimismo, es indispensable desarrollar una visión integral donde intervenga el análisis de condiciones, procesos y resultados.

Por otra parte, hay que mencionar que es necesaria la reflexión, tanto por parte de quienes planean como de aquellos que evalúan, acerca del carácter e intención que institucionalmente se confiere a este nivel de estudios, buscando que este ejercicio siente las bases para formar criterios acertados para el establecimiento, orientación y acción de las prioridades de oferta, frente al aumento de la demanda y la necesidad de cobertura de este tipo de servicios.

Ante este marco de retos por atender es necesario considerar lo siguiente:

- Aún cuando se ha dado un impresionante desarrollo en los diversos ámbitos del conocimiento, se presenta una escasa presencia de propuestas innovadoras en los aspectos académicoorganizativo. Cabe hacer la advertencia que, aquella modalidad de educación que emplea medios a distancia, reproduce los esquemas existentes; la única innovación es el medio que se emplea. 
- La creciente tendencia mundial de promover una orientación transdisciplinaria, capaz de proveer perspectivas novedosas en áreas de estudio, se contrapone con la visión de planeación académica de los posgrados en nuestro país -así como la formación y actualización- pues la tendencia es que éstos se desarrollen linealmente en un sólo campo disciplinario.

- Pese a los esfuerzos realizados en este renglón, procesos de toma de decisiones y de financiamiento que tienden al centralismo y a la reproducción de esquemas poco flexibles que derivan en una anquilosamiento de la oferta, aún persisten en el sistema educativo que atiende a este nivel.

- A pesar del Programa de Desarrollo del Posgrado, aún se muestra la tendencia histórica que denota la desigualdad en el progreso de este nivel educativo, manifestado tanto en montos de financiamiento, como en la oferta por zonas geográficas y por instituciones.

- La carencia de un diagnóstico real y objetivo sobre el impacto de la formación de los académicos de los posgrados en programas de cuarto nivel; las estadísticas denotan un aumento en la escolaridad de los cuerpos docentes de este tipo de programas, pero no evalúan la existencia y la acción eficiente de cuerpos académicos colegiados que funcionen como masa crítica.

El desarrollo de una perspectiva integral que atienda al contexto que se emplee para las tareas de planeación-evaluación del posgrado nacional, con criterios flexibles en su organización y administración académica, puede contribuir a conformar un marco que precise el sentido, las posibles acciones y la pertinencia de programas de esta naturaleza:

La creciente importancia de los factores ambientales de naturaleza económica, cultural y política en las organizaciones gubernamentales que generó, inclusive nuevos abordajes teóricos de la administración para el desarrollo, la ecología administrativa y el abordaje contigencial, dio origen a nuevos criterios definidores para los actos y hechos administrativos. Al lado de la racionalidad administrativa medida según los criterios técnicos de eficiencia y eficacia, surgen la efectividad y la relevancia como criterios esencialmente políticos y filosóficos de la administración. ${ }^{14}$

\section{PLANEACIÓN Y POSGRADO.}

Los estudios de posgrado ubicados en un marco de desarrollo nacional a mediano y largo plazo tienen un compromiso específico vinculado directamente a los aportes que pueden hacer quienes los cursan. Dado que uno de los objetivos es formar cuadros de alto nivel para el ejercicio de la docencia y la investigación, los beneficios de dicha responsabilidad quedan ubicados no sólo en lo que se refiere al desarrollo conceptual sino también en la contribución de métodos que coadyuven a resolver los problemas que enfrenta la sociedad mexicana.

Aún cuando existe cierto œnsenso en lo anterior, los criterios coyunturales siguen siendo los que orientan el establecimiento y desarrollo del posgrado; en muchos casos la improvisación es evidente, lo que se detecta en los resultados con dos indicadores claves: la escasa demanda, y una oferta educativa poco relevante y pertinente. Es necesario destacar la importancia de respetar las especificidades institucionales, y asentar los criterios metodológicos a atender con el fin de que la oferta resulte pertinente; para ello, al

\footnotetext{
${ }^{14}$ SANDER, B. (1982) p. 9-21
} 
establecer la factibilidad y pertinencia del programa se hace imprescindible incluir ciertos aspectos básicos: las condiciones diagnósticas del entorno institucional, el papel social (general) del programa, un estudio de la demanda potencial, así como un diagnóstico acerca del impacto en los sectores sociales mós afines al campo disciplinar del programa.

Es importante señalar que si no se realiza lo anterior, el programa no fracasará, pero dentro de las condiciones de cambio permanente en los entornos universitarios caracterizados por una gran inestabilidad financiera, un ejercicio serio de planeación resulta clave para crear condiciones idóneas para el surgimiento y consolidación del programa de posgrado bajo esquemas de calidad. No se propone una subordinación ala técnica administrativa, la idea es que la toma de decisiones académicas se sustente en ella con la finalidad de crear un marco prospectivo adecuado, permitiría alcanzar, en primera instancia, los objetivos educacionales; y en última instancia, los fines institucionales.

Es aquí donde se muestra la importancia de las herramientas y técnicas como auxiliares de las tareas académicas, cuyo propósito fundamental se sustenta en lo siguiente: la búsqueda y aplicación de criterios de calidad, tanto en la planeación institucional como en el desarrollo de las tareas de enseñanzaaprendizaje.

Desde esta perspectiva, las técnicas administrativas contribuyen a la configuración de un marco valorativo de calidad que apoye el esquema de trabajo, los objetivos y fines últimos de un programa de posgrado al interior de una universidad pública pero, que por sí mismo, no puede convertirse en un parámetro de calidad y pertinencia del programa al que deben ajustarse la totalidad de las actividades académicas, en este sentido: "Toda sociedad parece natural a sus miembros en la medida en que, por la adhesión a sus símbolos y por la confianza en sus padrones, sienten ellos la propia existencia como alguna cosa que armoniza con ese orden." ${ }^{15}$

En este escenario, es innegable el esfuerzo hecho recientemente, pero aún con todos los recursos no se ha superado un reto de origen: la visión de expansión de los posgrados, preferentemente en el sentido cuantitativo (como lo es el aumento del número de programas) que deja de lado la pertinencia dentro de un marco institucional y social.

Es claro que la actual capacidad instalada de las escuelas de posgrado, a pesar de su crecimiento relativamente rápido, es todavía muy pequeña en comparación con la magnitud de la matrícula y los requisitos cualitativos del personal docente de las universidades. Igualmente, todavía ésta es muy reducida y desequilibrada en relación con la investigación futura y los requerimientos de desarrollo de la sociedad y la economía mexicana. En años recientes, sólo el 3.3 por ciento del total de la matrícula de la educación superior, pública y privada, correspondía a los postgrados. ${ }^{16}$

\section{CONSECUENCIAS DEL USO AMBIGUO DE CONCEPTOS}

Los conceptos con los que se construye y expresa el mundo que nos rodea provienen, de una manera u otra, de ideas consensuadas socialmente; es decir, el concepto no existe por sí, sino por lo que significa para el grupo social que lo emplea:

\footnotetext{
${ }^{15}$ GUERREIRO RAMOS, A. ( 1981 ) p.126

${ }^{16}$ COOMBS, Ph. (1985) p.73
} 
Las realidades son también diferentes en función de la ciencia que les contemple. Un ser humano, es en si mismo, un cúmulo de realidades diferentes [...]. El ser humano es, pues, un compedio de realidades entendidas desde un contexto semántica, puesto que el lenguaje -los signos y las palabras que transcriben las ideas y los hechos constatados- determina la diferencia de lo que, en realidad, es una realidad única: la del ser humano. (...) El hombre vive inmerso en una idea semántica del Universo."17

Esto es, colocar un concepto en un contexto diferente de su origen, y buscar instruirlo sin un proceso de construcción de sentido acarrea diversas consecuencias, entre las que destacan: ambigüedad en las formas y fines, graves dificultades en la comunicación dentro de la organización; y finalmente, un rechazo a los procesos de cambio ligados al concepto central que $\cong$ impulsa, "[...] el hecho se condiciona al uso del lenguaje y al significado de los signos, siempre diferente en función del contexto en que se expresen". ${ }^{18}$

En el caso de las universidades públicas, el proceso de consulta y búsqueda de consenso de los conceptos que cohesionan a la institución, inicialmente parte, de una actividad organizacional y social conjunta de quienes la conforman -real o virtualmente- que les lleva, de manera u otra, a acceder la idea general y el sentido de la organización con determinados propósitos, fines y métodos. Este proceso significa algo más que la simple repetición y aceptación impensada, mecánica y lineal de una idea originada e instituida jerárquicamente; la gran diferencia radica en el proceso participativo que se da para la construcción del propio concepto, que, si bien no llega ser la panacea para las consecuencias enunciadas líneas arriba, puede contribuir hipotéticamente a resolverlas en tanto se atienden anticipadamente.

Esta práctica coadyuva al vencimiento de la noción burocrática que existe en muchas de las universidades del país apegadas a un esquema centralista. De manera insistente, no se trata de no evaluar sino de pensar cómo y con qué sentido se hace, sobre todo en atención a los objetivos institucionales y a la pertinencia social que ésta debe a su entorno.

\section{LÍNEAS DE CALIDAD PARA UN POSGRADO PERTINENTE.}

Con relación a la necesidad de la planeación para alcanzar la calidad y pertinencia, se proponen una serie de medidas que pueden contribuir a modificar la situación general, que también sirven de conclusión para el presente trabajo. Los criterios centrales para fijar las condiciones necesarias para el surgimiento y operación de los programas, entre otros, son los siguientes: que sus objetivos, programas y proyectos de desarrollo demuestren ser válidos para un horizonte de tiempo mayor a los cinco años, y de acuerdo con lo anterior, su estructura organizativa y los recursos necesarios para su operación se justifiquen con base en programas académicos de mediano y largo plazo.

A continuación se presentan una serie de anotaciones que pueden contribuir a desarrollar estratégicamente el posgrado, con la salvedad de las anotaciones sobre la participación que se sugieren:

a) Fuerte y sostenido impulso a la cultura de la planeación prospectiva.

b) Diversificación de fuentes de financiamiento; de tal manera que si la institución lo considera importante no se sujete sólo a las políticas oficiales que definen la relevancia de un programa y desmeritan la necesidad de otro.

\footnotetext{
${ }^{17}$ RACIONERO, L. (1990) p. 19

${ }^{18}$ RACIONERO, L. (1990) p.20
} 
c) Elaboración constante de diagnósticos del entorno para aseguramiento de la calidad y la pertinencia del programa.

d) Establecimiento y fortalecimiento de redes de cooperación efectivas a nivel nacional e internacional.

e) Consideración de las asimetrías regionales.

f) Construcción de nuevas filosofías de la acción del egresado (acción responsable y pertinente).

g) Visiones del conocimiento acordes a los tiempos (paradigma de complejidad).

h) Noción de acción para el desarrollo sustentable.

i) Vinculación con la investigación. Aún cuando se ha discutido la necesidad de relacionar incondicionalmente las actividades de docencia en el nivel posgrado y las tareas de investigación, es necesario analizar si esta condición por sí misma asegura la formación de elementos de alto nivel y con más calidad.

j) Líneas de prioridad. En este renglón lo que se deberá considerar es la regionalización de los programas, atendiendo en especial a las necesidades detectadas por medio de diagnósticos que se lleven a cabo con anticipación a la apertura del programa; además posibilitar la selectividad de acuerdo a criterios sociales e institucionales.

k) Coordinación y cooperación. Se considera clave la creación de redes orgánicas ínter e intra institucionales, con el fin de hacer común la información que se maneja para la planeación de los posgrados.

l) Vinculación con el contexto. Se convierte en un elemento fundamental para la planeación prospectiva del posgrado, pues permite la construcción social de la necesidad del tipo de estudios, así como de la orientación, y sobre todo, el criterio y la jerarquía de la respuesta académica que está dando la universidad.

La UNESCO ha descrito diez de las tendencias ${ }^{19}$ que necesariamente transformarán la sociedad así como los espacios educativos de todos los niveles, pero especialmente en el posgrado, donde se constituyen los marcos de la acción pertinente de este tipo de estudios, aspectos indispensables en la planeación. Éstas son:

Tabla 1.

Tendencias de desarrollo para el siglo XXI

\begin{tabular}{|c|c|c|}
\hline TENDENCIAS & $\begin{array}{c}\text { POSIBLE IMPACTO EN } \\
\text { EDUCACIÓN }\end{array}$ & IMPACTO EN POSGRADO \\
\hline
\end{tabular}

${ }^{19}$ UNESCO (2002). 


\begin{tabular}{|c|c|c|}
\hline $\begin{array}{l}\text { El auge de la tercera revolución } \\
\text { industrial. La tercera revolución } \\
\text { industrial que está transformando } \\
\text { radicalmente a las sociedades, se } \\
\text { manifiesta en el auge de la } \\
\text { revolución informática, el } \\
\text { desarrollo acelerado de las } \\
\text { ciencias y tecnologías de la } \\
\text { comunicación e información, y el } \\
\text { avance de la biología y la genética. }\end{array}$ & $\begin{array}{l}\text { Obsolescencia de conocimientos. } \\
\text { Limitación de aportes para la } \\
\text { resolución de problemas a partir } \\
\text { de la visión segmentada de la } \\
\text { ciencia (campos disciplinares vs. } \\
\text { transdisciplina). } \\
\text { Rezago en la infraestructura } \\
\text { informática. }\end{array}$ & $\begin{array}{l}\text { Necesidad de transformar el } \\
\text { sentido de la formación en ese } \\
\text { nivel, así como modificar los } \\
\text { campos de trabajo. }\end{array}$ \\
\hline $\begin{array}{l}\text { Agravación de la pobreza y la } \\
\text { exclusión. El aumento de la } \\
\text { pobreza no sólo se mide en } \\
\text { parámetros económicos, sino } \\
\text { también en términos educativos, } \\
\text { tecnológicos, culturales, } \\
\text { medioambientales y sanitarios. }\end{array}$ & $\begin{array}{l}\text { Aumento de población excluida } \\
\text { de servicios educativos. }\end{array}$ & $\begin{array}{l}\text { Crear estrategias que aporten } \\
\text { soluciones que coadyuven a } \\
\text { solucionar este reto de la } \\
\text { humanidad. } \\
\text { Necesidad de formar científicos } \\
\text { con una percepción más integral } \\
\text { de su quehacer (noción socio- } \\
\text { histórica). }\end{array}$ \\
\hline $\begin{array}{l}\text { Nuevas amenazas para la paz, la } \\
\text { seguridad y los derechos } \\
\text { humanos. El deterioro del medio } \\
\text { ambiente y de las condiciones de } \\
\text { vida, los problemas de población, } \\
\text { las rivalidades culturales y étnicas } \\
\text { y todas las formas de violación a } \\
\text { los derechos humanos. }\end{array}$ & $\begin{array}{l}\text { Transformación de las } \\
\text { prioridades gubernamentales } \\
\text { impactando las tareas educativas } \\
\text { en su sentido y forma. }\end{array}$ & $\begin{array}{l}\text { Necesidad de atender nuevos } \\
\text { campos del conocimiento. } \\
\text { Mayor vinculación entre ciencia y } \\
\text { tecnología. }\end{array}$ \\
\hline $\begin{array}{l}\text { Mutaciones demográficas. El } \\
\text { aumento de la población mundial } \\
\text { conlleva una urbanización masiva, } \\
\text { acelerada por las } \\
\text { transformaciones } \\
\text { socioeconómicas, que transforma } \\
\text { la escala de las ciudades y va } \\
\text { acompañada por fenómenos sin } \\
\text { precedentes: pobreza y exclusión } \\
\text { urbanas, secesión de grupos y } \\
\text { barrios, problemas ambientales, } \\
\text { acceso a los recursos naturales y } \\
\text { culturales, derecho a la vivienda, } \\
\text { nuevos problemas de ciudadanía y } \\
\text { retracción del espacio publico. }\end{array}$ & $\begin{array}{l}\text { Demanda de servicios educativos } \\
\text { bajo nuevos esquemas } \\
\text { operativos (didácticas, } \\
\text { metodológicas, financieras, etc.) }\end{array}$ & $\begin{array}{l}\text { Desequilibrio regional en el } \\
\text { desarrollo del nivel. }\end{array}$ \\
\hline $\begin{array}{l}\text { El medio ambiente del planeta en } \\
\text { peligro. }\end{array}$ & $\begin{array}{l}\text { Transformación de los planes y } \\
\text { programas. } \\
\text { Modificación de las prácticas de } \\
\text { operación de los centros } \\
\text { escolares. }\end{array}$ & $\begin{array}{l}\text { Apertura de nuevos campos de } \\
\text { formación y de investigación. }\end{array}$ \\
\hline $\begin{array}{l}\text { Avances de la sociedad de la } \\
\text { información. Aunque se está } \\
\text { dando un avance, éste es desigual } \\
\text { entre los países desarrollados y } \\
\text { los que están en vías de desarrollo } \\
\text { e incluso dentro de cada país por } \\
\text { regiones. } \\
\text { Mas allá de la innovación industrial } \\
\text { que aportan las nuevas } \\
\text { tecnologías de la información y la } \\
\text { comunicación, se perfilan distintos } \\
\text { modelos de sociedad. }\end{array}$ & $\begin{array}{l}\text { Acrecentamiento de la brecha } \\
\text { científica y tecnológica. }\end{array}$ & $\begin{array}{l}\text { Desequilibrio en la atención por } \\
\text { zonas, así como por áreas } \\
\text { problemáticas. }\end{array}$ \\
\hline Reforzar la gobernabilidad. & $\begin{array}{l}\text { Modelos innovadores para la } \\
\text { gestión educativa. }\end{array}$ & $\begin{array}{l}\text { Surgimiento de nuevas formas } \\
\text { de gestión. }\end{array}$ \\
\hline
\end{tabular}




\begin{tabular}{|c|c|c|}
\hline Hacia la igualdad de los sexos. & $\begin{array}{l}\text { Transformación de los } \\
\text { programas y procesos educativos } \\
\text { en contenidos y metodologías }\end{array}$ & $\begin{array}{l}\text { Transformación de los } \\
\text { programas y procesos } \\
\text { educativos en contenidos y } \\
\text { metodologías. } \\
\text { Crear condiciones materiales } \\
\text { para la participación de la mujer } \\
\text { en este nivel educativo. }\end{array}$ \\
\hline $\begin{array}{l}\text { Nuevos encuentros entre las } \\
\text { culturas. Impacto de las nuevas } \\
\text { tecnologías. }\end{array}$ & $\begin{array}{l}\text { Cambio en las prácticas } \\
\text { educativas. }\end{array}$ & $\begin{array}{l}\text { Establecimiento de trabajo vía } \\
\text { redes. }\end{array}$ \\
\hline $\begin{array}{l}\text { Desafíos éticos de la tecnociencia. } \\
\text { Los numerosos progresos de la } \\
\text { biotecnología, la genética y la } \\
\text { astrofísica, así como de las } \\
\text { ciencias de lo infinitamente grande } \\
\text { y pequeño, están revolucionando } \\
\text { nuestra percepción de los seres } \\
\text { vivos y del mundo que nos rodea. } \\
\text { Terapias génicas, uso de la eficaz } \\
\text { de la energía, biotecnologías } \\
\text { aplicadas a la agricultura. }\end{array}$ & $\begin{array}{l}\text { Reorientación del sentido de lo } \\
\text { educativo. }\end{array}$ & $\begin{array}{l}\text { Necesidad de crear nuevos } \\
\text { marcos para la acción científica } \\
\text { y tecnológica que impacten la } \\
\text { formación de los investigadores. }\end{array}$ \\
\hline
\end{tabular}

Las tendencias descritas traen aparejadas una serie de situaciones que de una $u$ otra forma deben enfrentar todas las organizaciones sociales, incluyendo las de carácter educativo. Así, tales situaciones se traducen en las siguientes tensiones ${ }^{20}$-sin ser nuevas en muchos casos-, que se encuentran en el centro de la problemática del siglo XXI:

Como una manera de argumentar lo anterior, se cita a la UNESCO, organismo que menciona que de cara a las circunstancias globales desconocidas, todas las organizaciones sociales -incluyendo las educativas- se verán enfrentadas a cuando menos diez tensiones ${ }^{21}$ que deberán atenderse para actuar con oportunidad; éstas son:

Tabla 2.

Tensiones en la problemática del siglo XXI.

\begin{tabular}{|l|l|}
\hline \multicolumn{1}{|c|}{ Tensión } & \multicolumn{1}{|c|}{ Significado } \\
\hline Lo mundial vs. Lo local. & $\begin{array}{l}\text { "convertirse poco a poco en ciudadano del mundo sin perder sus raíces y } \\
\text { participando activamente en la vida de la nación y las comunidades de } \\
\text { base". }\end{array}$ \\
\hline $\begin{array}{l}\text { Lo universal vs. lo } \\
\text { singular. }\end{array}$ & $\begin{array}{l}\text { "la mundialización de la cultura se realiza progresivamente pero todavía } \\
\text { parcialmente. De hecho, es inevitable con sus promesas y sus riesgos, } \\
\text { entre los cuales no es el menor el de olvidar el carácter único de cada } \\
\text { persona, su vocación de escoger su destino y realizar todo su potencial, en } \\
\text { la riqueza mantenida de sus tradiciones y de su propia cultura, } \\
\text { amenazada, si no se presta atención, por las evoluciones que se están } \\
\text { produciendo". }\end{array}$ \\
\hline Tradición vs. modernidad. & $\begin{array}{l}\text { "adaptarse sin negarse a sí mismo, edificar su autonomía en dialéctica } \\
\text { con la libertad y la evolución de los demás, dominar el progreso científico. } \\
\text { Con este ánimo conviene enfrentarse al desafío de las nuevas tecnologías } \\
\text { de la información". }\end{array}$ \\
\hline
\end{tabular}

\footnotetext{
${ }^{20}$ DELORS, J. (1997) pp.12-13

${ }^{21}$ Estas tensiones pueden ser entendidas también como retos o problemas a las que se ven sometidas las organizaciones por parte del entorno y a las que se deberá atender de forma pertinente y con calidad.
} 


\begin{tabular}{|c|c|}
\hline $\begin{array}{l}\text { La noción de largo plazo } \\
\text { vs. el corto plazo. }\end{array}$ & $\begin{array}{l}\text { "tensión eterna pero alimentada actualmente por un predominio de lo } \\
\text { efímero y de la instantaneidad, en un contexto en que la plétora de } \\
\text { informaciones y emociones fugaces conduce incesantemente a una } \\
\text { concentración en los problemas inmediatos. Las opiniones piden } \\
\text { respuestas y soluciones rápidas, mientras que muchos de los problemas } \\
\text { encontrados necesitan una estrategia paciente, concertada y negociada de } \\
\text { reforma. Tal es precisamente el caso de las políticas educativas". }\end{array}$ \\
\hline $\begin{array}{l}\text { La indispensable } \\
\text { competencia y la } \\
\text { preocupación por la } \\
\text { igualdad de } \\
\text { oportunidades. }\end{array}$ & $\begin{array}{l}\text { "Cuestión clásica, planteada desde comienzo de siglo a las políticas } \\
\text { económicas y sociales y a las políticas educativas; cuestión resuelta a } \\
\text { veces pero nunca en forma duradera. Hoy, la Comisión corre el riesgo de } \\
\text { afirmar que la presión de la competencia hace olvidar a muchos directivos } \\
\text { la misión de dar a cada ser humano los medios de aprovechar todas sus } \\
\text { oportunidades. Esta constatación nos ha conducido, en el campo que } \\
\text { abarca este informe, a retomar y actualizar el concepto de educación } \\
\text { durante toda la vida, para conciliar la competencia que estimula, la } \\
\text { cooperación que fortalece y la solidaridad que une". }\end{array}$ \\
\hline $\begin{array}{l}\text { El extraordinario desarrollo } \\
\text { de los conocimientos y las } \\
\text { capacidades } \\
\text { asimilación del de } \\
\text { humano. }\end{array}$ & $\begin{array}{l}\text { "añadir nuevas disciplinas como el conocimiento de sí mismo y los } \\
\text { medios de mantener la salud física y psicológica, o el aprendizaje para } \\
\text { conocer mejor el medio ambiente natural y preservarlo. Y sin embargo, los } \\
\text { programas escolares cada vez están más recargados. Por tanto, será } \\
\text { necesario escoger, en una clara estrategia de reforma, pero a condición de } \\
\text { preservar los elementos esenciales de una educación básica que enseñe } \\
\text { a vivir mejor mediante el conocimiento, la experimentación y la formación } \\
\text { de una cultura personal". }\end{array}$ \\
\hline $\begin{array}{l}\text { Lo espiritual y lo material, } \\
\text { que también es una } \\
\text { constatación eterna. }\end{array}$ & $\begin{array}{l}\text { "el mundo, frecuentemente sin sentirlo o expresarlo, tiene sed de ideal y de } \\
\text { valores que vamos a llamar morales para no ofender a nadie. ¡Que noble } \\
\text { tarea de la educación la de suscitar en cada persona, según sus } \\
\text { tradiciones y sus convicciones y con pleno respeto del pluralismo, esta } \\
\text { elevación del pensamiento y el espíritu hasta lo universal y a una cierta } \\
\text { superación de sí mismo! La supervivencia de la humanidad-la Comisión } \\
\text { lo dice midiendo las palabras- depende de ello". }\end{array}$ \\
\hline
\end{tabular}

Frente a todo lo descrito, se requiere un nuevo tipo de estudiante de posgrado $-\mathrm{y}$ de ciudadano-, el cual sólo podrá formarse en espacios innovadores, críticos, y que cuenten con una visión estratégica de la circunstancia actual, pero sin descartar la perspectiva para actuar en el mediano y el largo plazo. Si algo caracteriza a nuestro tiempo es la complejidad y la incertidumbre. ${ }^{22}$ Lo que nos lleva a un mundo complejo donde se requiere superar retos como la necesidad de atender lo inmediato y la necesidad de reflexionar para prever consecuencias.

Hoy, ya no se trata de ajustarse o adaptarse, porque ambas acciones suponen siempre un retraso. Hoy, de lo que se trata es de adelantarse. Hay que adoptar una visión a largo plazo de cara al porvenir y además, echar una mirada prospectiva al mundo para cosechar en el futuro los frutos de anticipación y no los problemas de una visión corta.

De ahí que, hablar de calidad en la formación de recursos humanos de alto nivel supone indispensablemente un proceso de planeación eficiente pero, no sólo desde la perspectiva de la misma labor de la gestión, sino desde la óptica de la discusión pedagógica-académica que construye sentidos para que todo quehacer institucional adquiera un rango de pertinencia, condición urgente en estos tiempos en un país como México.

\footnotetext{
${ }^{22}$ Morín, E. (2000).
} 
Lo anterior lleva a la inclusión necesaria de la propuesta que hace Morin ${ }^{23}$ respecto a aquellos saberes que debe poseer cualquier ciudadano del siglo XXI, y que de una u otra forma, es en los espacios educativos formales donde se está enfrentando la responsabilidad de impartir una educación que impulse estos saberes. En el caso de los estudios de posgrado, éstos deberán estar presentes en la construcción de sus sentidos y en los aspectos pedagógicos-operativos:

Tabla 3.

Saberes para el siglo XXI y su impacto social y educativo.

\begin{tabular}{|c|c|c|}
\hline SABERES & Impacto social & Impacto en el sistema educativo \\
\hline $\begin{array}{l}\text { Las cegueras del conocimiento: } \\
\text { el error y la ilusión }\end{array}$ & $\begin{array}{l}\text { Reflexión sobre el sentido de la } \\
\text { construcción de la ciencia. } \\
\text { Comprensión de las limitaciones } \\
\text { del conocimiento científico. "El } \\
\text { conocimiento del conocimiento } \\
\text { debe aparecer como una } \\
\text { necesidad primera que servirá de } \\
\text { preparación para enfrentar } \\
\text { riesgos permanentes de error e } \\
\text { ilusión que no cesan de parasitar } \\
\text { la mente humana." }\end{array}$ & $\begin{array}{l}\text { Transformación de los contenidos y } \\
\text { las metodologías empleadas en el } \\
\text { sistema. }\end{array}$ \\
\hline $\begin{array}{l}\text { Los principios del conocimiento } \\
\text { pertinente }\end{array}$ & $\begin{array}{l}\text { Transformación en la percepción } \\
\text { para incluir nociones globales } \\
\text { donde destaquen los } \\
\text { conocimientos locales. } \\
\text { Impulso al pensamiento holístico: } \\
\text { noción de integración de las } \\
\text { partes y las totalidades. } \\
\text { "Desarrollar una aptitud natural } \\
\text { de la inteligencia humana para } \\
\text { ubicar todas sus informaciones } \\
\text { en un contexto y en su conjunto. }\end{array}$ & $\begin{array}{l}\text { Desarrollo de nociones de } \\
\text { transdiciplinariedad para organizar } \\
\text { contenidos. } \\
\text { Revolución del pensamiento con la } \\
\text { intención del desarrollo de la } \\
\text { habilidad de contextualización } \\
\text { Enseñar los métodos que permitan } \\
\text { aprehender las relaciones mutuas y } \\
\text { las influencias reciprocas entre las } \\
\text { partes y el todo en un mundo } \\
\text { complejo. }\end{array}$ \\
\hline
\end{tabular}

\footnotetext{
${ }^{23}$ Morín E. (1999).
} 


\begin{tabular}{|c|c|c|}
\hline Enseñar la condición humana & $\begin{array}{l}\text { Transformación de los criterios } \\
\text { de acción de lo social a partir de } \\
\text { la consideración de lo humano } \\
\text { como punto de partida y llegada } \\
\text { de cada uno de los sentidos que } \\
\text { le motivan. } \\
\text { "Reconocer la la unidad y la } \\
\text { complejidad humanas reuniendo } \\
\text { y organizando conocimientos } \\
\text { dispersos en las ciencias de la } \\
\text { naturaleza, en las ciencias } \\
\text { humanas, la literatura y la filosofía } \\
\text { y mostrar la unión indisoluble } \\
\text { entre la unidad y la diversidad de } \\
\text { todo lo que es humano. }\end{array}$ & $\begin{array}{l}\text { Establecer como objeto esencial de } \\
\text { cualquier educación. }\end{array}$ \\
\hline Enseñar la identidad terrenal & $\begin{array}{l}\text { Comprensión de la ineludible } \\
\text { interrelación e interdependencia } \\
\text { entre quienes habitan este } \\
\text { planeta. } \\
\text { "Habrá que señalar la } \\
\text { complejidad de la crisis } \\
\text { planetaria que enmarca el siglo } \\
\text { XX mostrando que todos los } \\
\text { humanos, confrontados desde } \\
\text { ahora con los mismos problemas } \\
\text { de la vida y de la muerte, viven en } \\
\text { una misma comunidad de } \\
\text { destino." }\end{array}$ & $\begin{array}{l}\text { Modificación de los enfoque para } \\
\text { abordar las problemáticas } \\
\text { humanas. } \\
\text { Incorporación del nivel Kósmico } 24 \\
\text { como punto esencial de los análisis } \\
\text { actuales que se realizan dentro de } \\
\text { los contenidos educativos.. }\end{array}$ \\
\hline Enfrentar las incertidumbres & $\begin{array}{l}\text { Transformación acelerada en la } \\
\text { generación de los conocimientos, } \\
\text { impacto en los procesos } \\
\text { productivos. } \\
\text { "Las ciencias nos han hecho } \\
\text { adquirir muchas certezas, pero de } \\
\text { la misma manera nos han } \\
\text { revelado, en el siglo XX, } \\
\text { innumerables campos de } \\
\text { incertidumbre." }\end{array}$ & $\begin{array}{l}\text { "Se tendrían que enseñar } \\
\text { principios de estrategia que } \\
\text { permitan afrontar los riesgos, lo } \\
\text { inesperado, lo incierto, y modificar } \\
\text { su desarrollo en virtud de las } \\
\text { informaciones adquiridas en el } \\
\text { camino. Es necesario aprender a } \\
\text { navegar en océanos de } \\
\text { incertidumbres a través de } \\
\text { archipiélagos de certeza." }\end{array}$ \\
\hline Enseñar la comprensión & $\begin{array}{l}\text { Modificación de la perspectiva de } \\
\text { la otredad. } \\
\text { "La comprensión es al mismo } \\
\text { tiempo medio y fin de la } \\
\text { comunicación humana." } \\
\text { La comprensión mutua entre } \\
\text { humanos, tanto próximos como } \\
\text { extraños es en adelante vital para } \\
\text { que las relaciones humanas } \\
\text { salgan de su estado bárbaro de } \\
\text { incomprensión." }\end{array}$ & $\begin{array}{l}\text { Conformar líneas de trabajo sobre } \\
\text { educación para la paz. }\end{array}$ \\
\hline
\end{tabular}

\footnotetext{
${ }^{24}$ Wilber (2000:10) se refiere a este concepto: "Los griegos tenían una hermosa palabra - Kosmos -para referirse a la Totalidad ordenada de la existencia, una totalidad que incluía los reinos físicos, emocionales, mentales y espirituales. Desde su punto de vista, la realidad última, pues, no era tanto el cosmos (la dimensión estrictamente física) como el Kosmos (que incluye las dimensiones físicas, emocionales, mentales y espirituales). El Kosmos no se refería sólo a la materia inanimada e insensible, sino a la Totalidad viva compuesta por la materia, el cuerpo, la mente, el alma y el espíritu."
} 


\begin{tabular}{|l|l|l|}
\hline Ética del genero humano & $\begin{array}{l}\text { Incorporación de nuevos marcos } \\
\text { para la acción social. } \\
\text { "Concebir a la humanidad como } \\
\text { comunidad planetaria. }\end{array}$ & $\begin{array}{l}\text { La educación debe no solo } \\
\text { contribuir a una toma de conciencia } \\
\text { de nuestra Tierra-Patria, sino } \\
\text { también permitir que esta } \\
\text { conciencia se traduzca en la } \\
\text { voluntad de realizar la ciudadanía } \\
\text { terrenal." } \\
\text { "La ética no puede enseñarse con } \\
\text { lecciones de moral .Ella debe } \\
\text { formarse en las mentes a partir de } \\
\text { la conciencia de que el humano es } \\
\text { al mismo tiempo individuo, parte de } \\
\text { la sociedad y de la especie. }\end{array}$ \\
\hline
\end{tabular}

La voluntad de pertinencia se hace más urgente en las épocas de transformación social, de ahí que la UNESCO ${ }^{25}$ proponga lo siguiente: "Entonces más que nunca, la educación superior debe desempeñar un papel fundamental, poniendo todos sus recursos y su espíritu de independencia al servicio de lo que es pertinente para la persona y la sociedad en general [...]". Ser pertinente es estar en contacto:

- Con las políticas.

- $\quad$ Con el mundo del trabajo.

- Con la cultura y las culturas.

- Con todos.

- Siempre y en todas partes.

- Con los estudiantes y los profesores.

Luego entonces, considerar la posibilidad del calificativo de calidad dentro de un programa de posgrado requiere de la presencia del la pertinencia social sustentada en los elementos que se enuncian y sólidamente planeada, con el fin de que su impacto se vislumbre claramente en un escenario de futuro. Aquí lo que cabe es preguntarse, ¿se hizo?, ¿se ha hecho?, ¿se será capaz de realizarlo? Con lo anterior se propone que el concepto de calidad corresponde a una noción de cambio permanente construida cotidianamente a partir de una visión clara de futuro, y de la respuesta a las necesidades de los distintos sectores de la sociedad. Así se convierte en pieza clave a percepción del entorno social, por lo que un posgrado pertinente a los inicios del siglo XXI deberá contar con marcos de acción de amplios horizontes, tanto transversal, que permite captar las múltiples dimensiones de la vida humana asociada, como horizontal, que permite la percepción de escenarios a largo plazo.

\section{BIBLIOGRAFÍA}

ALBA, E. (1983). La investigación científica en las universidades ( tomo I) en: La crisis de la educación superior en México. México: Nueva Imagen (pp. 217-26).

ARNAZ, J. 1984) La planeación curricular. En Planeación de la educación superior. México: CONPES.

\footnotetext{
${ }^{25}$ UNESCO (1998). pp.19-27
} 
ASOCIACIÓN NACIONAL DE UNIVERSIDADES E INSTITUTOS DE EDUCACIÓN SUPERIOR (1979). La planeación de la educación superior. México: ANUIES.

ASOCIACIÓN NACIONAL DE UNIVERSIDADES E INSTITUTOS DE EDUCACIÓN SUPERIOR (2000). Educación Superior para el siglo XXI. México: ANUIES.

Bazúa, F., Valenti, G., Moya, L., y Villa García, L. (1993). Hacia un modelo alternativo de evaluación de los programas de posgrado en México. en : Universidad Futura. (vol. 5, núm.13, Invierno).

CAPRA, F. ( 1996 ). El punto crucial. México: Cuatro Estaciones.

CASILLAS, J. y HANEL, J. (1975). La creación de estudios de posgrado. En Revista de la Educación Superior. México: ANUIES (vol. IV, núm. 2, abril-julio, pp. 20-39).

Consejo nacional de Ciencia y TeCnología (2001). Programa especial de ciencia y tecnología. México: CONACYT.

COOMBS, Ph. (1985). La crisis mundial en la educación. Inglaterra: Oxford Press

COOMBS, Ph. (1991). Estrategia para mejorar la calidad de la educación superior en México. Informe para el secretario de Educación Pública realizado por el Consejo Internacional para el Desarrollo de la Educación. México: SEP-FCE

DELORS, J. (1997). La educación encierra un tesoro. México: UNESCO

GALLEGOS, R. (1996). Proyecto Quantum . Revista de la educación superior.

GARDNER, J. (1984). Excellence. London: NORTON Press.

MORIN, E. (1999). Los siete saberes necesarios a la educación del futuro. Paris: UNESCO.

MORIN, E. (2000). La mente bien ordenada España: Editorial Seix Barral.

MORIN, E. (2003). Educar en la era planetaria. Barcelona: GEDISA.

RACIONERO, L. y MEDINA, L. (1990). El nuevo paradigma. Barcelona: Promociones y Publicaciones Universitarias.

SANDER, B. (1982). Administracao da educacao no Brasil, evolucao do conhecimento. Fortaleza: UFC

SEYMOUR, D. (1993). Causing quality in higher education. Phoenix: ORIX Press.

SIMONE, R. (2001). La tercera fase. Formas de saber que estamos perdiendo. Madrid: TAURUS.

TEDESCO, J. C. (2000). Educar en la era del conocimiento. México: Fondo de Cultura Económica.

TUNNERMAN C. (2002). La calidad y pertinencia social en las instituciones de educación superior. Segundo Congreso Nacional y Tercero Internacional de Retos y expectativas de la Universidad. Toluca: Universidad Autónoma del Estado de México.

United Nations Education, Science Culture Organization (1996). La educación encierra un tesoro. Paris: UNESCO. 
UnIted Nations EdUCATION, SCIENCE CultuRE ORganization (1998). La educación superior en el siglo XXI: visión y acción. Paris: UNESCO.

UnItEd Nations Education, SCIENCE CULTURE ORganization (2000). Informe provisional del equipo especial sobre la UNESCO en el siglo XXI. En http://www. unesdoc.unesco.org/images/0011/001196/119699s.pdf

United Nations Education, Science Culture Organization (2002). Futuros posibles. Diez tendencias para el siglo XXI. En Nuevo Correo de la UNESCO. Mayo. Francia: UNESCO.

WILBER, K. (2000). Una teoría de todo. Barcelona: Kairós. 


\title{
Contactar
}

Revista lberoamericana de Educación

\author{
Principal OEI
}

